\title{
Radial BMD and serum CTX-I can Predict the Progression of Carotid Plaque in Rheumatoid Arthritis: A 3-year Prospective Cohort Study
}

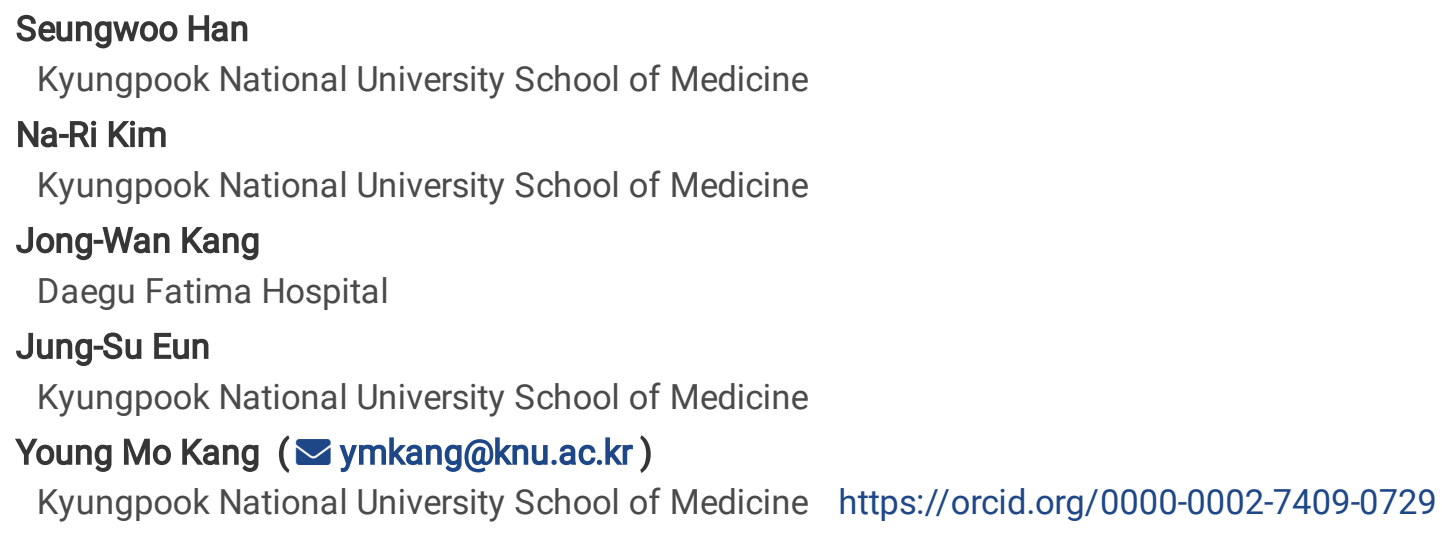

Research article

Keywords: Rheumatoid arthritis, Atherosclerosis, Risk factor, Bone mineral density, C-terminal telopeptide of type-I collagen Posted Date: January 29th, 2021

DOI: https://doi.org/10.21203/rs.3.rs-156810/v1

License: @) (1) This work is licensed under a Creative Commons Attribution 4.0 International License. Read Full License 


\section{Abstract}

Objective. Patients with rheumatoid arthritis (RA) are almost twice as likely to develop cardiovascular disease (CVD) as those without. However, traditional CVD risks have been shown to underperform in RA patients; thus, we aimed to identify new surrogate risk factors to better reflect their atherosclerotic burden.

Methods. A total of 380 RA patients with carotid atherosclerosis data were analyzed in this prospective cohort study. The primary outcome was carotid plaque progression over the 3-year follow-up period. Risk parameters assessed for the progression of carotid plaque were categorized as demographics, traditional CVD risks, RA-related risks, and bone parameters.

Results. The progression of carotid plaque was associated with the level of rheumatoid factor $(p=0.025)$, serum C-terminal telopeptide of type-I collagen (CTX-I) $(p=0.014)$, and femur and distal radius bone mass density (BMD) $(p=0.007$ and 0.004 , respectively), as well as traditional CVD risk factors. In multivariable analyses, the bone parameters of serum CTX-I and distal radius $\mathrm{BMD}$ proved to be independent predictors of the progression of carotid plaque along with hyperlipidemia, smoking, and baseline carotid plaque (all, $\mathrm{p}<0.05$ ). Adding both serum CTX-I and distal radius BMD increased the carotid plaque progression prediction model's percentage of explained variance from $24 \%$ to $30 \%$.

Conclusion. High serum CTX-I and lower radius BMD, reflecting high bone turnover, were independent risk factors for the progression of carotid plaque in RA patients, implicating the direct or indirect role of bone catabolism on the atherosclerotic burden.

\section{Introduction}

Patients with rheumatoid arthritis (RA) are nearly twice as likely to die before the age of 75 compared with people without the disease; the leading cause of this excess mortality among all age groups is cardiovascular disease (CVD) [1]. This is mainly mediated by the increased burden of atherosclerosis in RA patients. However, identifying RA patients at risk for CVD is challenging since the standard CVD risk stratification tools developed for the general population have been shown to underperform in RA patients [2]. Indeed, the Framingham risk equation for predicting CVD outcomes failed to predict almost half of the events that would occur in RA patients [2]. Thus, attempts have been made to establish a RA-specific CVD risk prediction equation [3, 4], but external validation has failed to uniformly predict the CVD risk in RA populations [4]. Moreover, traditional risk factors justify only about $20 \%$ of the variance in the total burden of subclinical atherosclerosis, even in the general population [5]. This highlights the need for a new risk surrogate that better reflects the atherosclerotic burden, especially in RA patients.

The cellular component of atherosclerotic lesions predominately comprises smooth muscle cells and macrophages [6]. Macrophages that surround calcium deposits can have an anti-inflammatory phenotype of the M2 macrophage, which could potentially display osteoclast (OC)-like function [7]. Actually, tartrate-resistant acid phosphatase (TRAP)-positive OC-like cells have been identified in atherosclerotic lesions, and the knockout of cathepsin $\mathrm{K}$, the OC-specific lysosomal protease, shows an attenuated atherosclerotic progression, suggesting that OCs play a role in the pathogenesis of atherosclerosis [8, 9]. In addition, atherosclerotic lesions have osteoblast-like cells, which are transdifferentiated from vascular endothelial cells, smooth muscle cells, and mesenchymal cell-like fibroblasts [10]. These bone phenotype cells play a critical role in the pathogenesis of atherosclerosis, which is mainly induced by inflammation followed by vascular wall mineralization [11]. Along with these basic and translational studies, recent clinical reports also suggest that decreased bone mineral density (BMD) is an independent predictor for CVD [12].

To investigate the risk factors for CVD and its contribution to mortality in RA patients, we established the Kyungpook National University Hospital (KNUH) Atherosclerosis Risk in RA (KARRA) cohort; wherein we observed that the presence of carotid plaque in RA patients was associated with a cumulative inflammatory burden, such as the cumulative erythrocyte sedimentation rate (ESR) and health assessment questionnaire (HAQ) score, as well as current disease activity, including the Disease activity score-28 using ESR (DAS28-ESR) [13]. However, those results were drawn-out under the study's cross-sectional design; 
consequently, it was hard to conclude the causal and temporal relationships between the risks and carotid plaque. The aim of this 3-year prospective, longitudinal cohort study was identification of a novel surrogate risk factor to predict the progression of subclinical atherosclerosis in RA patients and, in particular, to evaluate whether bone-related variables are associated with the atherosclerosis burden.

\section{Patients And Methods}

\section{Study design and Patients: the KARRA cohort}

During the period from September 2009 to November 2011, 417 consecutive RA patients, who fulfilled the 1987 American College of Rheumatology Classification for RA, were recruited from the rheumatology clinic of KNUH to establish the KARRA cohort for a prospective, longitudinal, observation study [13]. At baseline, carotid ultrasound examinations were performed in 380 patients, 309 of whom also underwent the 3-year follow-up carotid ultrasound (Fig. 1). Ethical approval was obtained from KNUH Institutional Review Board (reference number: KNUH_09-1054), and written informed consent was obtained from all participants.

\section{Carotid ultrasound to assess carotid intima-media thickness (IMT) and plaque}

The primary outcome in this study was the progression of carotid plaque by carotid ultrasound examination after the 3-year follow-up period. Carotid ultrasound examination was conducted using high-resolution B-mode images acquired with LOGIQ7 ultrasound system (General Electric, Milwaukee, WI) with a 10-MHz linear transducer by one qualified ultrasound technician. Carotid plaque was defined as either a focal structure that encroaches into the arterial lumen by at least $50 \%$ of the surrounding (intima-media thickness) IMT value, or a thickness of $>1.5 \mathrm{~mm}$ as measured from the media-adventitia interface to the intima-lumen interface. The progression of carotid plaque was defined as an increase in the number of carotid plaques at the 3-year follow-up ultrasound compared to that at baseline. The carotid IMT was measured using continuous static images, 20$\mathrm{mm}$ in length, obtained from 10-mm below the proximal common carotid artery through the middle of the internal carotid artery [13]. The mean and maximal IMT values were automatically analyzed with dedicated software (Intimascope TM, Media Cross, Japan).

\section{Demographic, clinical variables and laboratory tests}

Demographic and clinical parameters of CVD- and RA-associated risk factors were obtained by experienced rheumatologists and clinical research nurses (Table 1). At baseline, body-mass index (BMI) was calculated as the weight in kilograms divided by the square of height in meters. Waist-Hip ratio (WHR) was determined by dividing the waist circumference at the level of the navel by the maximum hip circumference. We diagnosed hypertension, diabetes, and hypercholesterolemia if the resting blood pressure was persistently above $140 / 90 \mathrm{mmHg}$, the fasting plasma glucose was $\geq 126 \mathrm{mg} / \mathrm{dL}$ or the non-fasting plasma glucose level was $\geq 200 \mathrm{mg} / \mathrm{dL}$, and the serum low-density lipoprotein cholesterol level was $\geq 130 \mathrm{mg} / \mathrm{dL}$, respectively, or if a person was receiving treatment for any of the above. Smoking status was classified as never-smoker, former smoker who had smoked > 100 cigarettes in their lifetime, and current-smoker. For RA-related risk factors, the disease duration was assessed by asking the date when the first symptoms of synovitis began, and a modified HAQ survey was conducted to assess the functional status in RA patients. DAS-28 with ESR (DAS28-ESR) was used to assess the activity status of RA: DAS28-ESR scores $<2.6,2.6-3.2,3.21-5.1$, and $>5.1$ were interpreted as remission, mild, moderate, and severe disease activity, respectively. 
Table 1

Baseline demographic, clinical characteristics and laboratory findings according to the progression of carotid plaque after 3year follow-up of the KARRA cohort

\begin{tabular}{|c|c|c|c|c|c|c|c|c|}
\hline \multirow[t]{2}{*}{ Baseline parameters } & \multicolumn{4}{|c|}{ Total cohort $(n=309)$} & \multicolumn{4}{|c|}{ No carotid plaque at baseline $(n=169)$} \\
\hline & $\begin{array}{l}\text { No } \\
\text { progression } \\
(n=171)\end{array}$ & $\begin{array}{l}\text { Progression } \\
(n=138)\end{array}$ & $\mathbf{P}$ & $\begin{array}{l}\text { OR } \\
(95 \% \mathrm{Cl})\end{array}$ & $\begin{array}{l}\text { No } \\
\text { plaque } \\
(n= \\
120)\end{array}$ & $\begin{array}{l}\text { New } \\
\text { plaque } \\
(n=49)\end{array}$ & $\mathbf{P}$ & $\begin{array}{l}\text { OR } \\
(95 \% \mathrm{Cl})\end{array}$ \\
\hline Male (\%) & $21(12.3)$ & $33(23.9)$ & 0.010 & $\begin{array}{l}2.245 \\
(1.230- \\
4.096)\end{array}$ & $11(9.2)$ & $9(12.2)$ & 0.546 & $\begin{array}{l}1.383 \\
(0.481- \\
3.973)\end{array}$ \\
\hline Age (yr) & $\begin{array}{l}53.06 \pm \\
11.67\end{array}$ & $\begin{array}{l}60.34 \pm \\
9.47\end{array}$ & $<.001$ & $\begin{array}{l}1.065 \\
(1.041- \\
1.089)\end{array}$ & $\begin{array}{l}48.78 \pm \\
9.95\end{array}$ & $\begin{array}{l}56.49 \pm \\
9.09\end{array}$ & $\hat{0} .001$ & $\begin{array}{l}1.083 \\
(1.043- \\
1.125)\end{array}$ \\
\hline \multicolumn{9}{|l|}{ CV risk factors } \\
\hline $\mathrm{BMI}\left(\mathrm{kg} / \mathrm{m}^{2}\right)$ & $\begin{array}{l}22.78 \pm \\
3.58\end{array}$ & $\begin{array}{l}23.14 \pm \\
2.82\end{array}$ & 0.331 & $\begin{array}{l}1.035 \\
(0.966- \\
1.109)\end{array}$ & $\begin{array}{l}22.69 \pm \\
3.73\end{array}$ & $\begin{array}{l}23.02 \pm \\
2.69\end{array}$ & 0.572 & $\begin{array}{l}0.991 \\
(0.957- \\
1.026)\end{array}$ \\
\hline Waist-hip ratio & $0.89 \pm 0.07$ & $0.92 \pm 0.06$ & 0.002 & $\begin{array}{l}276.9 \\
(7.017- \\
10929.1)\end{array}$ & $\begin{array}{l}0.88 \pm \\
0.07\end{array}$ & $\begin{array}{l}0.90 \pm \\
0.06\end{array}$ & 0.052 & $\begin{array}{l}154.4 \\
(0.959- \\
24860.9)\end{array}$ \\
\hline Hypertension & $37(21.6)$ & $43(31.2)$ & 0.068 & $\begin{array}{l}1.639 \\
(0.982- \\
2.736)\end{array}$ & $\begin{array}{l}20 \\
(16.7)\end{array}$ & $\begin{array}{l}13 \\
(26.5)\end{array}$ & 0.142 & $\begin{array}{l}1.806 \\
(0.815- \\
4.000)\end{array}$ \\
\hline Diabetes Mellitus & $8(4.7)$ & $12(8.7)$ & 0.169 & $\begin{array}{l}1.940 \\
(0.770- \\
4.890)\end{array}$ & $3(2.5)$ & $3(6.1)$ & 0.248 & $\begin{array}{l}2.543 \\
(0.495- \\
13.063)\end{array}$ \\
\hline Hypercholesterolemia & $14(8.2)$ & $26(18.8)$ & 0.006 & $\begin{array}{l}2.603 \\
(1.301- \\
5.208)\end{array}$ & $9(7.5)$ & $8(16.3)$ & 0.083 & $\begin{array}{l}2.407 \\
(0.870- \\
6.657)\end{array}$ \\
\hline Smoking: ever \& current & $21(12.3)$ & $38(27.5)$ & 0.001 & $\begin{array}{l}2.714 \\
(1.505- \\
4.896)\end{array}$ & $\begin{array}{l}15 \\
(12.5)\end{array}$ & $8(16.3)$ & 0.510 & $\begin{array}{l}1.366 \\
(0.538- \\
3.465)\end{array}$ \\
\hline Ever & $12(7.0)$ & 19 (13.8) & 0.058 & $\begin{array}{l}2.116 \\
(0.989- \\
4.527)\end{array}$ & $8(6.7)$ & $6(12.2)$ & 0.233 & $\begin{array}{l}1.953 \\
(0.640- \\
5.960)\end{array}$ \\
\hline Current & $9(5.3)$ & 19 (13.8) & 0.015 & $\begin{array}{l}2.874 \\
(1.256- \\
6.575)\end{array}$ & $7(5.8)$ & $2(4.1)$ & 0.645 & $\begin{array}{l}0.687 \\
(0.138- \\
3.429)\end{array}$ \\
\hline $\begin{array}{l}\text { LDL-cholesterol } \\
(\mathrm{mg} / \mathrm{dL})\end{array}$ & $\begin{array}{l}109.61 \pm \\
29.65\end{array}$ & $\begin{array}{l}116.78 \pm \\
32.69\end{array}$ & 0.046 & $\begin{array}{l}1.007 \\
(1.000- \\
1.015)\end{array}$ & $\begin{array}{l}105.89 \\
\pm 28.04\end{array}$ & $\begin{array}{l}120.14 \\
\pm 32.55\end{array}$ & 0.005 & $\begin{array}{l}1.016 \\
(1.004- \\
1.028)\end{array}$ \\
\hline Uric acid (mg/dL) & $3.67 \pm 1.35$ & $4.00 \pm 1.47$ & 0.041 & $\begin{array}{l}1.183 \\
(1.005- \\
1.392)\end{array}$ & $\begin{array}{l}3.59 \pm \\
1.18\end{array}$ & $\begin{array}{l}3.59 \pm \\
1.19\end{array}$ & 0.974 & $\begin{array}{l}0.995 \\
(0.750- \\
1.321)\end{array}$ \\
\hline $\begin{array}{l}\text { Presence of carotid } \\
\text { plaque }\end{array}$ & $51(29.8)$ & $89(64.5)$ & $<.001$ & $\begin{array}{l}4.274 \\
(2.649- \\
6.896)\end{array}$ & NA & NA & NA & NA \\
\hline
\end{tabular}

Abbreviations: CV; Cardiovascular, BMl; Body-mass index, LDL; Low-density lipoprotein, MHAQ; Modified Health assessment questionnaire, DAS28; Disease activity score 28, ESR; Erythrocyte sedimentation rate, CRP; C-reactive protein, ACPA; Anticitrullinated protein antibody, TNF; Tumor necrosis factor, CTX-I; C-terminal telopeptides type I collagen, BMD; Bone mineral density, OR; Odds ratio, Cl; Confidence interval, NA; not available 


\begin{tabular}{|c|c|c|c|c|c|c|c|c|}
\hline \multirow[t]{2}{*}{ Baseline parameters } & \multicolumn{4}{|c|}{ Total cohort $(n=309)$} & \multicolumn{4}{|c|}{ No carotid plaque at baseline $(n=169)$} \\
\hline & $\begin{array}{l}\text { No } \\
\text { progression } \\
(n=171)\end{array}$ & $\begin{array}{l}\text { Progression } \\
(n=138)\end{array}$ & $\mathbf{P}$ & $\begin{array}{l}\text { OR } \\
(95 \% \mathrm{Cl})\end{array}$ & $\begin{array}{l}\text { No } \\
\text { plaque } \\
(n= \\
120)\end{array}$ & $\begin{array}{l}\text { New } \\
\text { plaque } \\
(n=49)\end{array}$ & $\mathbf{P}$ & $\begin{array}{l}\text { OR } \\
(95 \% \mathrm{Cl})\end{array}$ \\
\hline Mean carotid IMT, mm & $0.76 \pm 0.15$ & $0.88 \pm 0.17$ & $<.001$ & $\begin{array}{l}187.9 \\
(33.2- \\
1064.0)\end{array}$ & $\begin{array}{l}0.72 \pm \\
0.13\end{array}$ & $\begin{array}{l}0.81 \pm \\
0.12\end{array}$ & $<.001$ & $\begin{array}{l}256.6 \\
(16.5- \\
3991.9)\end{array}$ \\
\hline \multicolumn{9}{|l|}{$\begin{array}{l}\text { RA-associated risk } \\
\text { factor }\end{array}$} \\
\hline Disease duration (yr) & $\begin{array}{l}12.21 \pm \\
7.89\end{array}$ & $\begin{array}{l}13.74 \pm \\
8.83\end{array}$ & 0.112 & $\begin{array}{l}1.022 \\
(0.995- \\
1.051)\end{array}$ & $\begin{array}{l}11.28 \pm \\
6.28\end{array}$ & $\begin{array}{l}12.23 \pm \\
6.91\end{array}$ & 0.392 & $\begin{array}{l}1.023 \\
(0.972- \\
1.076)\end{array}$ \\
\hline mHAQ & $6.19 \pm 7.11$ & $7.52 \pm 6.63$ & 0.095 & $\begin{array}{l}1.028 \\
(0.995- \\
1.063)\end{array}$ & $\begin{array}{l}5.31 \pm \\
5.91\end{array}$ & $\begin{array}{l}7.43 \pm \\
6.74\end{array}$ & 0.044 & $\begin{array}{l}1.053 \\
(1.000- \\
1.109)\end{array}$ \\
\hline DAS28-ESR & $3.21 \pm 1.12$ & $3.41 \pm 1.26$ & 0.123 & $\begin{array}{l}1.162 \\
(0.960- \\
1.406)\end{array}$ & $\begin{array}{l}3.18 \pm \\
1.13\end{array}$ & $\begin{array}{l}3.26 \pm \\
1.11\end{array}$ & 0.676 & $\begin{array}{l}1.065 \\
(0.793- \\
1.432)\end{array}$ \\
\hline $\operatorname{ESR}(\mathrm{mm} / \mathrm{h})$ & $\begin{array}{l}23.91 \pm \\
20.08\end{array}$ & $\begin{array}{l}28.39 \pm \\
21.28\end{array}$ & 0.059 & $\begin{array}{l}1.011 \\
(0.999- \\
1.022)\end{array}$ & $\begin{array}{l}23.81 \pm \\
20.80\end{array}$ & $\begin{array}{l}25.75 \pm \\
17.77\end{array}$ & 0.570 & $\begin{array}{l}1.005 \\
(0.988- \\
1.021)\end{array}$ \\
\hline $\mathrm{CRP}(\mathrm{mg} / \mathrm{dL})$ & $0.39 \pm 0.68$ & $0.53 \pm 1.14$ & 0.177 & $\begin{array}{l}1.201 \\
(0.906- \\
1.593)\end{array}$ & $\begin{array}{l}0.41 \pm \\
0.76\end{array}$ & $\begin{array}{l}0.41 \pm \\
0.64\end{array}$ & 0.983 & $\begin{array}{l}1.005 \\
(0.634- \\
1.593)\end{array}$ \\
\hline $\begin{array}{l}\text { Rheumatoid factor } \\
(\mathrm{IU} / \mathrm{mL})\end{array}$ & $\begin{array}{l}61.03 \pm \\
80.77\end{array}$ & $\begin{array}{l}90.22 \pm \\
131.96\end{array}$ & 0.025 & $\begin{array}{l}1.003 \\
(1.000- \\
1.005)\end{array}$ & $\begin{array}{l}56.76 \pm \\
55.17\end{array}$ & $\begin{array}{l}67.85 \pm \\
109.67\end{array}$ & 0.347 & $\begin{array}{l}1.002 \\
(0.998- \\
1.006)\end{array}$ \\
\hline ACPA (Unit/mL) & $\begin{array}{l}285.34 \pm \\
217.48\end{array}$ & $\begin{array}{l}296.17 \pm \\
234.73\end{array}$ & 0.699 & $\begin{array}{l}1.000 \\
(0.999- \\
1.001)\end{array}$ & $\begin{array}{l}290.94 \\
\pm 218.68\end{array}$ & $\begin{array}{l}267.48 \\
\pm 224.36\end{array}$ & 0.565 & $\begin{array}{l}1.000 \\
(0.998- \\
1.001)\end{array}$ \\
\hline Steroid $\geq 5 \mathrm{mg}$ & $20(11.9)$ & $13(9.4)$ & 0.486 & $\begin{array}{l}0.770 \\
(0.368- \\
1.609)\end{array}$ & $\begin{array}{l}13 \\
(10.9)\end{array}$ & $4(8.2)$ & 0.509 & $\begin{array}{l}0.725 \\
(0.224- \\
2.344)\end{array}$ \\
\hline $\begin{array}{l}\text { Methotrexate dosage } \\
\text { (mg/wk) }\end{array}$ & $\begin{array}{l}10.77 \pm \\
3.87\end{array}$ & $\begin{array}{l}11.28 \pm \\
3.31\end{array}$ & 0.242 & $\begin{array}{l}1.040 \\
(0.973- \\
1.111)\end{array}$ & $\begin{array}{l}10.85 \pm \\
3.9\end{array}$ & $\begin{array}{l}11.06 \pm \\
3.08\end{array}$ & 0.751 & $\begin{array}{l}1.016 \\
(0.923- \\
1.118)\end{array}$ \\
\hline TNF inhibitors & $10(5.8)$ & $9(6.5)$ & 0.806 & $\begin{array}{l}1.123 \\
(0.443- \\
2.846)\end{array}$ & $6(5.0)$ & $2(4.1)$ & 0.799 & $\begin{array}{l}0.809 \\
(0.157- \\
4.151)\end{array}$ \\
\hline \multicolumn{9}{|l|}{ Bone parameter } \\
\hline Osteocalcin (ng/mL) & $7.17 \pm 3.29$ & $7.71 \pm 3.55$ & 0.170 & $\begin{array}{l}1.048 \\
(0.979- \\
1.122)\end{array}$ & $\begin{array}{l}7.12 \pm \\
2.70\end{array}$ & $\begin{array}{l}8.39 \pm \\
4.09\end{array}$ & 0.021 & $\begin{array}{l}1.126 \\
(1.014- \\
1.251)\end{array}$ \\
\hline Serum CTX-I (pg/mL) & $0.37 \pm 0.19$ & $0.42 \pm 0.24$ & 0.014 & $\begin{array}{l}3.972 \\
(1.288- \\
12.253)\end{array}$ & $\begin{array}{l}0.36 \pm \\
0.18\end{array}$ & $\begin{array}{l}0.44 \pm \\
0.22\end{array}$ & 0.014 & $\begin{array}{l}8.171 \\
(1.446- \\
46.154)\end{array}$ \\
\hline \multicolumn{9}{|l|}{ BMD mean $\left(\mathrm{g} / \mathrm{cm}^{2}\right)$} \\
\hline $\begin{array}{l}\text { Abbreviations: CV; Card } \\
\text { questionnaire, DAS28; } \\
\text { citrullinated protein ant } \\
\text { density, OR; Odds ratio, }\end{array}$ & $\begin{array}{l}\text { scular, BMI } \\
\text { se activity } \\
\text {, TNF; Tun } \\
\text { onfidence }\end{array}$ & $\begin{array}{l}\text { dy-mass inc } \\
\text { re 28, ESR; } \\
\text { ecrosis fac } \\
\text { val, NA; not }\end{array}$ & $\begin{array}{l}\text { LDL; L } \\
\text { irocyte } \\
\text { CTX-l; } \\
\text { ilable }\end{array}$ & $\begin{array}{l}\text { I-density } \\
\text { ediment } \\
\text { erminal }\end{array}$ & $\begin{array}{l}\text { rotein, m } \\
\text { rate, CRP } \\
\text { eptides ty }\end{array}$ & $\begin{array}{l}\text {; Modifi } \\
\text { eactive } \\
\text { collage }\end{array}$ & $\begin{array}{l}\text { Health } \\
\text { ein, A } \\
\text { MD; B }\end{array}$ & $\begin{array}{l}\text { A; Anti- } \\
\text { e mineral }\end{array}$ \\
\hline
\end{tabular}




\begin{tabular}{|c|c|c|c|c|c|c|c|c|}
\hline \multirow[t]{2}{*}{ Baseline parameters } & \multicolumn{4}{|c|}{ Total cohort $(n=309)$} & \multicolumn{4}{|c|}{ No carotid plaque at baseline $(n=169)$} \\
\hline & $\begin{array}{l}\text { No } \\
\text { progression } \\
(n=171)\end{array}$ & $\begin{array}{l}\text { Progression } \\
(n=138)\end{array}$ & $\mathbf{P}$ & $\begin{array}{l}\text { OR } \\
(95 \% \mathrm{Cl})\end{array}$ & $\begin{array}{l}\text { No } \\
\text { plaque } \\
(n= \\
120)\end{array}$ & $\begin{array}{l}\text { New } \\
\text { plaque } \\
(n=49)\end{array}$ & $\mathbf{P}$ & $\begin{array}{l}\text { OR } \\
\text { (95\% Cl) }\end{array}$ \\
\hline Lumbar BMD & $1.06 \pm 0.19$ & $1.03 \pm 0.21$ & 0.187 & $\begin{array}{l}0.436 \\
(0.127- \\
1.496)\end{array}$ & $\begin{array}{l}1.07 \pm \\
0.17\end{array}$ & $\begin{array}{l}1.03 \pm \\
0.20\end{array}$ & 0.154 & $\begin{array}{l}0.207 \\
(0.024- \\
1.818)\end{array}$ \\
\hline Femur BMD & $0.86 \pm 0.14$ & $0.82 \pm 0.16$ & 0.007 & $\begin{array}{l}0.106 \\
(0.020- \\
0.554)\end{array}$ & $\begin{array}{l}0.87 \pm \\
0.13\end{array}$ & $\begin{array}{l}0.82 \pm \\
0.14\end{array}$ & 0.026 & $\begin{array}{l}0.045 \\
(0.003- \\
0.719)\end{array}$ \\
\hline Radius BMD & $0.60 \pm 0.12$ & $0.55 \pm 0.14$ & 0.004 & $\begin{array}{l}0.059 \\
(0.008- \\
0.421)\end{array}$ & $\begin{array}{l}0.61 \pm \\
0.11\end{array}$ & $\begin{array}{l}0.56 \pm \\
0.13\end{array}$ & 0.010 & $\begin{array}{l}0.019 \\
(0.001- \\
0.426)\end{array}$ \\
\hline \multicolumn{9}{|l|}{$\begin{array}{l}\text { Osteopenia or } \\
\text { osteoporosis (T-score< } \\
-1.0 \text { ) }\end{array}$} \\
\hline Lumbar & $71(45.5)$ & $73(60.8)$ & 0.015 & $\begin{array}{l}1.859 \\
(1.147- \\
3.015)\end{array}$ & $\begin{array}{l}48 \\
(43.6)\end{array}$ & $\begin{array}{l}25 \\
(61.0)\end{array}$ & 0.058 & $\begin{array}{l}2.018 \\
(0.971- \\
4.196)\end{array}$ \\
\hline Femur & $67(39.2)$ & $39(50.0)$ & 0.065 & $\begin{array}{l}1.552 \\
(0.986- \\
2.443)\end{array}$ & $\begin{array}{l}40 \\
(36.7)\end{array}$ & $\begin{array}{l}24 \\
(58.5)\end{array}$ & 0.016 & $\begin{array}{l}2.435 \\
(1.170- \\
5.070)\end{array}$ \\
\hline Radius & $70(45.5)$ & $80(69.6)$ & $\begin{array}{l}< \\
0.001\end{array}$ & $\begin{array}{l}2.743 \\
(1.650- \\
4.560)\end{array}$ & $\begin{array}{l}41 \\
(37.6)\end{array}$ & $\begin{array}{l}24 \\
(60.0)\end{array}$ & 0.015 & $\begin{array}{l}2.488 \\
(1.185- \\
5.224)\end{array}$ \\
\hline \multicolumn{9}{|c|}{$\begin{array}{l}\text { Abbreviations: CV; Cardiovascular, BMI; Body-mass index, LDL; Low-density lipoprotein, mHAQ; Modified Health assessment } \\
\text { questionnaire, DAS28; Disease activity score 28, ESR; Erythrocyte sedimentation rate, CRP; C-reactive protein, ACPA; Anti- } \\
\text { citrullinated protein antibody, TNF; Tumor necrosis factor, CTX-I; C-terminal telopeptides type I collagen, BMD; Bone mineral } \\
\text { density, OR; Odds ratio, Cl; Confidence interval, NA; not available }\end{array}$} \\
\hline
\end{tabular}

Morning venipuncture blood samples were obtained after a 12-hour fast and were analyzed in KNUH clinical laboratories. Blood tests for metabolic diseases (fasting glucose, lipid profile, insulin resistance, and serum uric acid) and RA (ESR, CRP, rheumatoid factor and anti-citrullinated peptide antibody (ACPA)) were checked. Serum osteocalcin and C-terminal telopeptide of type-I collagen (CTX-I) was determined by automated radioimmunoassay and the electro-chemiluminescence method, respectively. BMD was evaluated at the lumbar spine, femoral neck, and distal radius by dual-energy X-ray absorptiometry using a Discovery W densitometer (Hologic ${ }^{\circledR}$, Marlborough, MA). BMD values were expressed in absolute values $\left(\mathrm{g} / \mathrm{cm}^{2}\right)$ and Tscores, which defines scores between -1 and -2.5 as osteopenia and scores below -2.5 as osteoporosis.

\section{Statistical analyses}

The association with the primary end point of carotid plaque progression was compared using a Student $t$ test for continuous variables and with the chi-square test for discrete parameters. The odds ratio (OR) for continuous variables was assessed by logistic regression with a single continuous variable.

Multivariable analyses was performed in 4 models by binary logistic regression analysis, with forward selection of the variables that demonstrated a statistical significance of P-value $<0.1$ in univariate analyses. The first model included only clinical variables as the covariate, such as age, male sex, WHR, hypertension, hypercholesterolemia, smoking status, serum LDLcholesterol, uric acid level, presence of baseline carotid plaque, mHAQ, RF, and ESR. The second and third model added the bone parameters of serum CTX-1 and distal radius BMD, respectively, and the fourth model included both bone parameters to clinical variables. The goodness of fit of each model was determined with the Nagelkerke $\mathrm{R}^{2}$ coefficient of determination. All statistical analyses were performed with the statistical package software IBM SPSS $®$ for Windows version 25. 


\section{Results}

\section{Progression of carotid plaque in 3-year interval and its risk factors.}

The 3-year follow-up carotid ultrasound performed on these 309 RA patients revealed the development of new plaque in 49 of the 169 patients without baseline carotid plaque, and the progression of plaque in 89 of the 140 patients with baseline plaque. Consequently, a total of 138 patients (44.7\%) demonstrated carotid plaque progression, but the other 171 patients did not (Fig. 1).

When we compared the baseline demographic and clinical characteristics of the total cohort, the following factors were significantly associated with the progression of carotid plaque in the univariate models: male sex, age, WHR, hypercholesterolemia, smoking, LDL-cholesterol, serum uric acid level, and the presence of baseline carotid plaque. Among the RA-related factors, only the level of rheumatoid factor was associated with the progression of carotid plaque after 3 years ( $p=$ $0.025)$, although the current ESR was marginally associated $(p=0.059)$. On the other hand, the parameters for disease activity (DAS-28 ESR), disease duration, or medications, including steroid, MTX, or biologics, failed to predict the progression of carotid plaque at the 3-year follow-up (Table 1, Supplementary Table 1, Supplementary Fig. 2). Among bone-related factors, the patients with a higher serum CTX-I level, and a lower femur and radius BMD had a higher incidence of carotid plaque progression (Table 1, Supplementary Fig. 1).

We then conducted a subgroup analysis on the 169 RA patients who did not have carotid plaque at baseline. The development of new carotid plaque was common in elderly patients and in those with higher LDL-cholesterol and mean carotid IMT at baseline. There was no significant association between the RA-associated risk factors and the development of new carotid plaque. The patients with an increased level of serum osteocalcin and CTX-1 at baseline had a higher incidence of carotid plaque development at the 3-year follow-up. In addition, lower baseline femur and radius BMDs were also significant risk factors for the development of carotid plaque at the 3-year follow-up (Table 1, Supplementary Fig. 1).

\section{Parameters reflecting bone turnover as an independent predictor of subclinical atherosclerosis.}

To identify the independent baseline risk factor for carotid plaque progression, variables with statistical significance in univariate analyses were subjected to backward stepwise multiple logistic regression analysis. When the variables associated with traditional CVD risk factors and RA-related factors were analyzed in model 1, the progression of carotid plaque was independently associated with the patient's age, hypercholesterolemia, smoking, and the presence of carotid plaque at baseline. These 4 variables explained $24.3 \%$ of the variance of this model $\left(R^{2}\right)$ for carotid plaque progression. Then, we added the bone parameters, CTX-I and distal radius BMD, to clinical models 2 and 3, respectively. The serum CTX-I and distal radius BMD remained as independent risk factor and were the most important predictors of carotid plaque progression among those evaluated. These parameters could increase the explained variance of the model $\left(R^{2}\right)$ to $27.3 \%$ and $25.6 \%$, respectively. Adding both CTX-I and distal radius BMD to the clinical risk factors in model 4 could explain $29.7 \%$ of the variance in the regression model for predicting carotid plaque progression (Table 2). 
Table 2

Regression coefficients and OR $(95 \% \mathrm{Cl})$ of best models based on backwards regression analysis for the progression of carotid plaque over 3 years in RA patients

\begin{tabular}{|c|c|c|c|c|c|c|c|c|}
\hline \multirow[b]{2}{*}{ Baseline parameters* } & \multicolumn{2}{|c|}{$\begin{array}{l}\text { Model 1: Clinical } \\
\text { factors }(n=252)\end{array}$} & \multicolumn{2}{|c|}{$\begin{array}{l}\text { Model 2: CTX-I }(n= \\
\text { 244) }\end{array}$} & \multicolumn{2}{|c|}{$\begin{array}{l}\text { Model 3: BMD radius } \\
(n=223)\end{array}$} & \multicolumn{2}{|c|}{$\begin{array}{l}\text { Model 4: CTX-I+ BMD } \\
\text { radius }(n=216)\end{array}$} \\
\hline & $\begin{array}{l}\beta \\
\text { coefficient } \\
\text { (SE) }\end{array}$ & $\begin{array}{l}\text { OR } \\
(95 \% \\
\mathrm{Cl})\end{array}$ & $\begin{array}{l}\beta \\
\text { coefficient } \\
\text { (SE) }\end{array}$ & $\begin{array}{l}\text { OR } \\
(95 \% \\
\mathrm{Cl})\end{array}$ & $\begin{array}{l}\beta \\
\text { coefficient } \\
(\mathrm{SE})\end{array}$ & $\begin{array}{l}\text { OR } \\
(95 \% \\
\mathrm{Cl})\end{array}$ & $\begin{array}{l}\beta \\
\text { coefficient } \\
\text { (SE) }\end{array}$ & $\begin{array}{l}\mathrm{OR}(95 \% \\
\mathrm{Cl})\end{array}$ \\
\hline Age & $\begin{array}{l}0.036 \\
(0.014)\end{array}$ & $\begin{array}{l}1.037 \\
(1.010- \\
1.065) \ddagger\end{array}$ & $\begin{array}{l}0.040 \\
(0.014)\end{array}$ & $\begin{array}{l}1.041 \\
(1.012- \\
1.070) \ddagger\end{array}$ & - & - & - & - \\
\hline Hypercholesterolemia & $\begin{array}{l}0.896 \\
(0.381)\end{array}$ & $\begin{array}{l}2.450 \\
(1.162- \\
5.164) \dagger\end{array}$ & $\begin{array}{l}1.024 \\
(0.393)\end{array}$ & $\begin{array}{l}2.784 \\
(1.290- \\
6.010) \ddagger\end{array}$ & $\begin{array}{l}0.963 \\
(0.390)\end{array}$ & $\begin{array}{l}2.621 \\
(1.221- \\
5.624) \dagger\end{array}$ & $\begin{array}{l}1.099 \\
(0.404)\end{array}$ & $\begin{array}{l}3.002 \\
(1.360- \\
6.626) \ddagger\end{array}$ \\
\hline $\begin{array}{l}\text { Smoking: ever \& } \\
\text { current }\end{array}$ & $\begin{array}{l}0.611 \\
(0.212)\end{array}$ & $\begin{array}{l}1.843 \\
(1.217- \\
2.791) \ddagger\end{array}$ & $\begin{array}{l}0.649 \\
(0.216)\end{array}$ & $\begin{array}{l}1.913 \\
(1.252- \\
2.924) \dagger\end{array}$ & $\begin{array}{l}0.903 \\
(0.261)\end{array}$ & $\begin{array}{l}2.467 \\
(1.480- \\
4.111) \ddagger\end{array}$ & $\begin{array}{l}0.988 \\
(0.272)\end{array}$ & $\begin{array}{l}2.687 \\
(1.577- \\
4.579) \S\end{array}$ \\
\hline $\begin{array}{l}\text { Presence of carotid } \\
\text { plaque }\end{array}$ & $\begin{array}{l}0.992 \\
(0.288)\end{array}$ & $\begin{array}{l}2.697 \\
(1.534- \\
4.739) \ddagger\end{array}$ & $\begin{array}{l}1.002 \\
(0.295)\end{array}$ & $\begin{array}{l}2.723 \\
(1.527- \\
4.853) \ddagger\end{array}$ & $\begin{array}{l}1.291 \\
(0.282)\end{array}$ & $\begin{array}{l}3.637 \\
(2.095- \\
6.316) \S\end{array}$ & $\begin{array}{l}1.353 \\
(0.291)\end{array}$ & $\begin{array}{l}3.869 \\
(2.190- \\
6.838) \S\end{array}$ \\
\hline Serum CTX-I (pg/mL) & NA & NA & $\begin{array}{l}1.539 \\
(0.667)\end{array}$ & $\begin{array}{l}4.662 \\
(1.262- \\
17.219)\end{array}$ & NA & NA & $\begin{array}{l}1.718 \\
(0.731)\end{array}$ & $\begin{array}{l}5.574 \\
(1.331- \\
23.341) \dagger\end{array}$ \\
\hline BMD radius $\left(\mathrm{g} / \mathrm{cm}^{2}\right)$ & NA & NA & NA & NA & $\begin{array}{l}-3.057 \\
(1.193)\end{array}$ & $\begin{array}{l}0.047 \\
(0.005- \\
0.488) \dagger\end{array}$ & $\begin{array}{l}-3.667 \\
(1.250)\end{array}$ & $\begin{array}{l}0.026 \\
(0.002- \\
0.296) \ddagger\end{array}$ \\
\hline $\mathrm{R}^{2}(\%)$ & 24.3 & & 27.3 & & 25.6 & & 29.7 & \\
\hline \multicolumn{9}{|c|}{$\begin{array}{l}\text { *Excluded variables which has statistical significance of } p<0.10 \text { for the progression of carotid plaque in univariate analysis } \\
\text { were as follows: sex, waist-hip ratio, the presence of hypertension, serum LDL-cholesterol level, serum uric acid level, mHAQ, } \\
\text { RF and ESR. }\end{array}$} \\
\hline \multicolumn{9}{|c|}{$\dagger p<0.05 ; \neq p<0.01 ; \S p<0.001 . R^{2}:$ Nagelkerke $R$ square } \\
\hline $\begin{array}{l}\text { Abbreviations: } \beta \text {; Partia } \\
\text { telopeptides type I colla }\end{array}$ & n, BMD; $B$ & miner & $\begin{array}{l}\text { Standard e } \\
\text { nsity, NA; n }\end{array}$ & $\begin{array}{l}\mathrm{r}, \mathrm{OR} ; \mathrm{O} \\
\text { availab }\end{array}$ & $\begin{array}{l}\text { ratio, } \mathrm{Cl} ; \mathrm{C} \\
{ }^{2} ; \text { Coeffici }\end{array}$ & $\begin{array}{l}\text { lence } \\
\text { f dete }\end{array}$ & $\begin{array}{l}\text { al, CTX-I; } \\
\text { tion }\end{array}$ & minal \\
\hline
\end{tabular}

Synergistic interactions between serum CTX-I and radius BMD in subclinical atherosclerosis.

The incidence of plaque progression was compared according to the serum CTX-I and radius BMD score to clarify the interaction between these two variables. Patients in the upper tertile of radius BMD $\left(\geq 0.625 \mathrm{~g} / \mathrm{cm}^{2}\right)$ showed only about $30 \%$ of the plaque progression incidence as the middle and lower tertile groups of serum CTX-I, and it increased to $37.5 \%$ in the upper CTX-I tertile group. In the middle tertile group of radius BMD $\left(0.530-0.625 \mathrm{~g} / \mathrm{cm}^{2}\right)$, the incidence of carotid plaque progression was gradually increased by the serum CTX-I level, that is $25.0 \%, 34.5 \%$ and $45.7 \%$ in the lower, middle, and upper CTX-I tertile groups, respectively. In the lower tertile group of radius BMD $\left(<0.530 \mathrm{~g} / \mathrm{cm}^{2}\right)$, plaque progression was observed in $42 \%$ of the patients in the lower tertile of serum CTX-I, and in nearly $60-70 \%$ of the patient in middle and upper tertiles (Fig. 2).

\section{Discussion}

We investigated the association of clinical, RA-related, and bone parameters for the progression of atherosclerosis by measuring the number of carotid plaques over 3-year interval in a 380-patient RA cohort. This study identifies a prospective and longitudinal relationship between the progression of carotid atherosclerosis and lower distal radius BMD and higher serum CTX-I levels at baseline, which reflects bone resorption and osteoclast activity. 
A number of epidemiologic studies have reported an association between subclinical atherosclerosis and BMD, not only in general population, but also in patients with inflammatory disease [14-17]. A Norwegian population-based study found a twofold increase in echogenic carotid plaque in subjects in the highest quartile of distal forearm BMD compared to those in the lowest quartile, while the presence of echolucent plaque was not associated with distal forearm BMD [14]. A lupus case-control study reported a two-fold increase in echolucent carotid plaque and a four-fold increase in echogenic plaque in patients with a lower hip BMD T-score of < - 1.0 standard deviation compared to controls [15]. Furthermore, the lupus patients in the lowest and middle tertiles of hip BMD were found to have about a three-fold increase in the carotid plaque index compared to the patients in the highest tertile of hip BMD, but the mean carotid IMT did not differ among the hip BMD tertiles [16]. More recently, a study of patients with psoriatic arthritis found an association between the presence of carotid plaque and total vertebral BMD, and suggested a link between interleukin-33 and its decoy receptor, soluble ST2, as an pathophysiologic mediator [17]. Overall, the studies to date have confirmed that a lower BMD is associated with the carotid plaque burden as a surrogate for subclinical atherosclerosis.

Another interesting finding of this study was that carotid plaque progressed faster in patients with a higher baseline CTX-1 level. To our knowledge, this is the first study to demonstrate the connection between atherosclerosis and CTX-1, the serum marker for osteoclast activity. There are two kinds of carboxy-terminal cross-linked telopeptide of type-I collagen: ICTP and CTX-1. Type-I collagen is degraded by the matrix metalloproteinase-2, $-9,-13$, or -14 , which produces ICTP, and then cathepsin $\mathrm{K}$ degrades the ICTP epitope, which produces CTX-1 [18]. As cathepsin K is produced mainly by activated macrophages and osteoclasts, the serum level of CTX-1 reflects the activity of macrophages and osteoclasts [19]. Actually, an atherosclerotic plaque lesion has form cells that originate from a macrophage lineage, which can also produce cathepsin $\mathrm{K}$, and it can increase the levels of CTX-1 through degradation of the atherosclerotic plaque $[19,20]$. However, it seems unlikely that this local proteolytic process can affect the serum CTX-1 level, considering the bone mass of the human body [20]. In our data, the upper tertile group of the distal radius BMD was not affected by the baseline serum CTX-1 level, showing only $30 \%$ of the progression of carotid plaque over the 3-year period. On the other hand, plaque progression in the middle and lower tertile groups was significantly affected by the baseline serum CTX-1 level (Fig. 1). This suggests that serum CTX-1 is closely connected to bone catabolism, rather than plaque remodeling itself.

The mechanisms connecting the atherosclerotic burden and enhanced bone catabolism are still poorly understood, but there are several potential hypotheses. The main effector cells in atherosclerosis and bone catabolism are foam cells and osteoclasts, respectively, and these two cells commonly originate from the monocyte-macrophage lineage [21]. Inflammatory cytokines, such as tumor necrosis factor-a and interleukin-6, can enhance the differentiation of the macrophage, and are responsible for the interaction between bone loss and atherosclerosis in RA patients [22]. Another possibility is the presence of a common mediator between atherosclerosis and osteoporosis, such as osteoprotegerin (OPG) or omentin-1. As a decoy receptor for RANKL, OPG can be elevated in the osteoporosis patient [23,24] and this increased OPG can suppress apoptosis of endothelial and vascular smooth muscle cells as a decoy receptor for TRAIL, leading to atherosclerosis [25-27]. In addition, omentin-1, a novel visceral adipose tissue-derived cytokine, is known to attenuate both bone loss and the progression of atherosclerosis [28, 29]. Finally, increased bone catabolism itself can be responsible for the association between osteoporosis and atherosclerosis. Evidence from bisphosphonate studies has shown that the inhibition of osteoclast function with bisphosphonate has an anti-atherosclerotic effect in both the normal population and lupus patients [30, 31]. Considering bisphosphonate accumulates mainly in bone by binding to hydroxyapatite crystals [32], the decreased bone catabolism by bisphosphonate and the consequent reduction of calcium-phosphate efflux or serum osteocalcin, an osteoblast-derived bone Gla ( $\gamma$-carboxyglutamic acid-containing) protein, can attenuate the progression of atherosclerosis [33, 34].

Contrary to our expectation, the disease activity of RA at baseline assessed by DAS28-ESR did not affect the progression of carotid plaque as well as the mean cIMT (Table 1, supplementary Fig. 3 and supplementary table 1). The 3-year prospective GIRRCS study on 841 Italian RA patients revealed that patients in the remission state of DAS28-ESR $(<2.6)$ at baseline had a significantly lower subclinical atherosclerotic lesion on their carotid or peripheral artery compared to active RA patients after 3 years [35]. Given our contrasting findings, this negative correlation of disease activity with atherosclerosis progression may result from the well-controlled RA activity in our study population. In fact, the mean baseline DAS28-ESR in our study was 3.2 
and 3.4 in plaque non-progression and progression groups, respectively. A prospective, observational study showed if the RA activity is well-controlled to remission or low activity (DAS28-ESR $\leq 3.2$ ) during a 3-year follow-up period, the risk of atherosclerosis progression is even similar to a non-RA control group [36]. Although our negative result may derive from the weak power to detect a small difference in this study, the RA activity does not seem to have a significant effect on the progression of atherosclerosis in patients whose RA disease activity is relatively well-controlled.

In conclusion, we investigated the potential risk for the progression of carotid plaque in RA patient in this prospective, observational study. The bone parameters of lower distal radius BMD and elevated serum CTX-1 level, along with hyperlipidemia, smoking, and the presence of carotid plaque at baseline were independent risk factors for the progression of atherosclerosis in RA patients. Our study sheds light on the connection between bone catabolism and atherosclerosis. Further clinical investigation is needed to determine whether this connection is RA-specific or a common phenomenon in the general population.

\section{Declarations}

\section{Ethics Declarations}

\section{Ethics approval and consent to participate}

Ethical approval was obtained from KNUH Institutional Review Board (reference number: KNUH_09-1054), and written informed consent was obtained from all participants.

\section{Consent for publication}

All authors agreed to the publication of this manuscript.

\section{Availability of data and material}

The data that support the findings of this study are available from the corresponding author, YM Kang, upon reasonable request.

\section{Competing interests}

The authors have declared no conflicts of interest.

\section{Funding}

This study was supported by a grant of the Korea Healthcare Technology R\&D Project, Ministry of Health and Welfare, Republic of Korea (A102065), and by a grant of BioMedical Research Institute KNUH 2010.

\section{Author contributions}

Study conception and design: S.H, N.K, J.K, J.E, Y.K. Acquisition of clinical data: N.K, J.K, J.E, Y.K. Analysis and interpretation of data: S.H, Y.K.

Drafting the article or revising it critically for important intellectual content: S.H, N.K, J.K, J.E, Y.K.

Final approval of the version of the article to be published: S.H, N.K, J.K, J.E, Y.K.

\section{Acknowledgments}

We thank Ga-Young Choi for performing carotid Doppler ultrasound examination for intima-media thickness and plaque measurement, as well as Sook-Kyung Park and Young-Ji Kim for conducting patient interviews and scheduling appointments. 


\section{References}

1. Widdifield J, Paterson JM, Huang A, Bernatsky S. Causes of Death in Rheumatoid Arthritis: How Do They Compare to the General Population? Arthritis Care Res (Hoboken). 2018;70(12):1748-55.

2. Crowson CS, Matteson EL, Roger VL, Therneau TM, Gabriel SE. Usefulness of risk scores to estimate the risk of cardiovascular disease in patients with rheumatoid arthritis. Am J Cardiol. 2012;110(3):420-4.

3. Solomon DH, Greenberg J, Curtis JR, et al. Derivation and internal validation of an expanded cardiovascular risk prediction score for rheumatoid arthritis: a Consortium of Rheumatology Researchers of North America Registry Study. Arthritis Rheumatol. 2015;67(8):1995-2003.

4. Crowson CS, Gabriel SE, Semb AG, et al. Rheumatoid arthritis-specific cardiovascular risk scores are not superior to general risk scores: a validation analysis of patients from seven countries. Rheumatology. 2017;56(7):1102-10.

5. Kuo F, Gardener H, Dong C, et al. Traditional cardiovascular risk factors explain the minority of the variability in carotid plaque. Stroke. 2012;43(7):1755-60.

6. Tsukada T, Rosenfeld M, Ross R, Gown AM. Immunocytochemical analysis of cellular components in atherosclerotic lesions. Use of monoclonal antibodies with the Watanabe and fat-fed rabbit. Arteriosclerosis. 1986;6(6):601-13.

7. Chinetti-Gbaguidi G, Daoudi M, Rosa M, et al. Human Alternative Macrophages Populate Calcified Areas of Atherosclerotic Lesions and Display Impaired RANKL-Induced Osteoclastic Bone Resorption Activity. Circ Res. 2017;121(1):19-30.

8. Qiao JH, Mishra V, Fishbein MC, Sinha SK, Rajavashisth TB. Multinucleated giant cells in atherosclerotic plaques of human carotid arteries: Identification of osteoclast-like cells and their specific proteins in artery wall. Exp Mol Pathol.

2015;99(3):654-62.

9. Lutgens $\mathrm{E}$, Lutgens $\mathrm{SP}$, Faber BC, et al. Disruption of the cathepsin $\mathrm{K}$ gene reduces atherosclerosis progression and induces plaque fibrosis but accelerates macrophage foam cell formation. Circulation. 2006;113(1):98-107.

10. Hortells L, Sur S, St Hilaire C. Cell Phenotype Transitions in Cardiovascular Calcification. Front Cardiovasc Med. $2018 ; 5: 27$.

11. Lampropoulos CE, Papaioannou I, D'Cruz DP. Osteoporosis-a risk factor for cardiovascular disease? Nat Rev Rheumatol. 2012;8(10):587-98.

12. Ye C, Xu M, Wang S, et al. Decreased Bone Mineral Density Is an Independent Predictor for the Development of Atherosclerosis: A Systematic Review and Meta-Analysis. PLoS One. 2016;11(5):e0154740.

13. Im CH, Kim NR, Kang JW, et al. Inflammatory burden interacts with conventional cardiovascular risk factors for carotid plaque formation in rheumatoid arthritis. Rheumatology. 2015;54(5):808-15.

14. Jorgensen L, Joakimsen O, Rosvold Berntsen GK, Heuch I, Jacobsen BK. Low bone mineral density is related to echogenic carotid artery plaques: a population-based study. Am J Epidemiol. 2004;160(6):549-56.

15. Ajeganova S, Gustafsson T, Jogestrand T, Frostegard J, Hafstrom I. Bone mineral density and carotid atherosclerosis in systemic lupus erythematosus: a controlled cross-sectional study. Arthritis Res Ther. 2015;17:84.

16. Ramsey-Goldman R, Manzi S. Association of osteoporosis and cardiovascular disease in women with systemic lupus erythematosus. Arthritis Rheum. 2001;44(10):2338-41.

17. Shen J, Shang Q, Wong CK, et al. Carotid plaque and bone density and microarchitecture in psoriatic arthritis: the correlation with soluble ST2. Sci Rep. 2016;6:32116.

18. Garnero P, Ferreras M, Karsdal MA, et al. The type I collagen fragments ICTP and CTX reveal distinct enzymatic pathways of bone collagen degradation. J Bone Miner Res. 2003;18(5):859-67.

19. Hakala JK, Oksjoki R, Laine P, et al. Lysosomal enzymes are released from cultured human macrophages, hydrolyze LDL in vitro, and are present extracellularly in human atherosclerotic lesions. Arterioscler Thromb Vasc Biol. 2003;23(8):1430-6.

20. Barascuk N, Skjot-Arkil H, Register TC, et al. Human macrophage foam cells degrade atherosclerotic plaques through cathepsin K mediated processes. BMC Cardiovasc Disord. 2010;10:19.

21. Rahman MS, Murphy AJ, Woollard KJ. Effects of dyslipidaemia on monocyte production and function in cardiovascular disease. Nat Rev Cardiol. 2017;14(7):387-400. 
22. Ait-Oufella H, Taleb S, Mallat Z, Tedgui A. Recent advances on the role of cytokines in atherosclerosis. Arterioscler Thromb Vasc Biol. 2011;31(5):969-79.

23. Mansour A, Aboeerad M, Qorbani M, et al. Association between low bone mass and the serum RANKL and OPG in patients with nephrolithiasis. BMC Nephrol. 2018;19(1):172.

24. Kim CS, Bae EH, Ma SK, et al. Association of Serum Osteoprotegerin Levels with Bone Loss in Chronic Kidney Disease: Insights from the KNOW-CKD Study. PLoS One. 2016;11(11):e0166792.

25. Emery JG, McDonnell P, Burke MB, et al. Osteoprotegerin is a receptor for the cytotoxic ligand TRAIL. J Biol Chem. 1998;273(23):14363-7.

26. Candido R, Toffoli B, Corallini F, et al. Human full-length osteoprotegerin induces the proliferation of rodent vascular smooth muscle cells both in vitro and in vivo. J Vasc Res. 2010;47(3):252-61.

27. Kiechl S, Schett G, Wenning G, et al. Osteoprotegerin is a risk factor for progressive atherosclerosis and cardiovascular disease. Circulation. 2004;109(18):2175-80.

28. Xie H, Xie PL, Wu XP, et al. Omentin-1 attenuates arterial calcification and bone loss in osteoprotegerin-deficient mice by inhibition of RANKL expression. Cardiovasc Res. 2011;92(2):296-306.

29. Hiramatsu-Ito M, Shibata R, Ohashi K, et al. Omentin attenuates atherosclerotic lesion formation in apolipoprotein Edeficient mice. Cardiovasc Res. 2016;110(1):107-17.

30. Caffarelli C, Montagnani A, Nuti R, Gonnelli S. Bisphosphonates, atherosclerosis and vascular calcification: update and systematic review of clinical studies. Clin Interv Aging. 2017;12:1819-28.

31. Ohmura $\mathrm{K}$, Kato $\mathrm{M}$, Watanabe $\mathrm{T}$, et al. Effect of combined treatment with bisphosphonate and vitamin $\mathrm{D}$ on atherosclerosis in patients with systemic lupus erythematosus: a propensity score-based analysis. Arthritis Res Ther. 2018;20(1):72.

32. Monkkonen J, Ylitalo P. The tissue distribution of clodronate (dichloromethylene bisphosphonate) in mice. The effects of vehicle and the route of administration. Eur J Drug Metab Pharmacokinet. 1990;15(3):239-43.

33. Foley RN, Collins AJ, Ishani A, Kalra PA. Calcium-phosphate levels and cardiovascular disease in community-dwelling adults: the Atherosclerosis Risk in Communities (ARIC) Study. Am Heart J. 2008;156(3):556-63.

34. Kapustin AN, Shanahan CM. Osteocalcin: a novel vascular metabolic and osteoinductive factor? Arterioscler Thromb Vasc Biol. 2011;31(10):2169-71.

35. Ruscitti P, Cipriani P, Liakouli V, et al. Subclinical and clinical atherosclerosis in rheumatoid arthritis: results from the 3-year, multicentre, prospective, observational GIRRCS (Gruppo Italiano di Ricerca in Reumatologia Clinica e Sperimentale) study. Arthritis Res Ther. 2019;21(1):204.

36. Arida A, Protogerou AD, Konstantonis G, Fragiadaki K, Kitas GD, Sfikakis PP. Atherosclerosis is not accelerated in rheumatoid arthritis of low activity or remission, regardless of antirheumatic treatment modalities. Rheumatology. 2017;56(6):934-9.

\section{Figures}




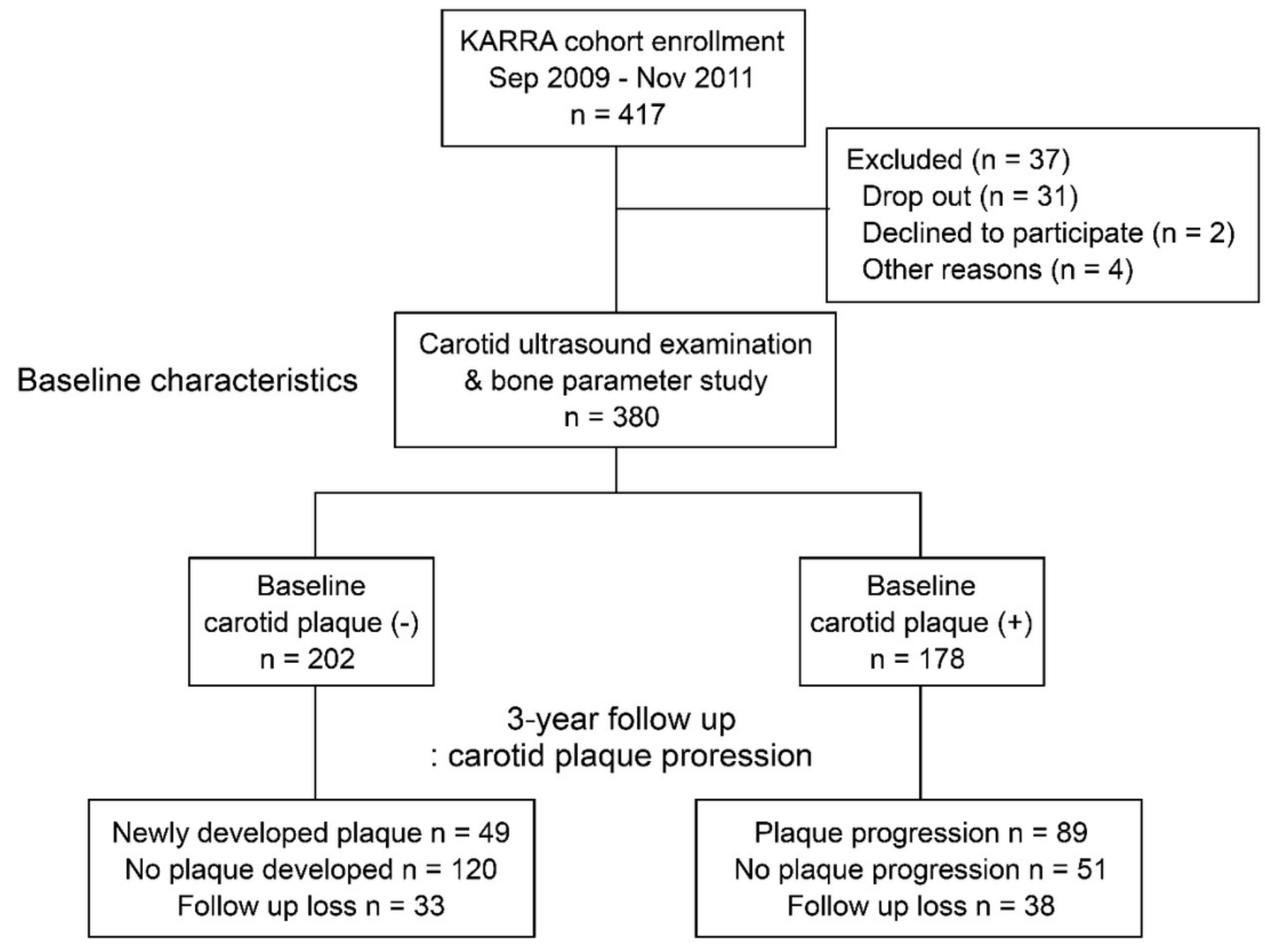

Figure 1

Flow chart of the KRAAR cohort study. Of the 417 RA patients recruited, we performed carotid ultrasound on 380 patients at baseline, and 3-year follow-up carotid ultrasound was performed in 309 patients. KARRA: KNUH Atherosclerosis Risk of Rheumatoid Arthritis 


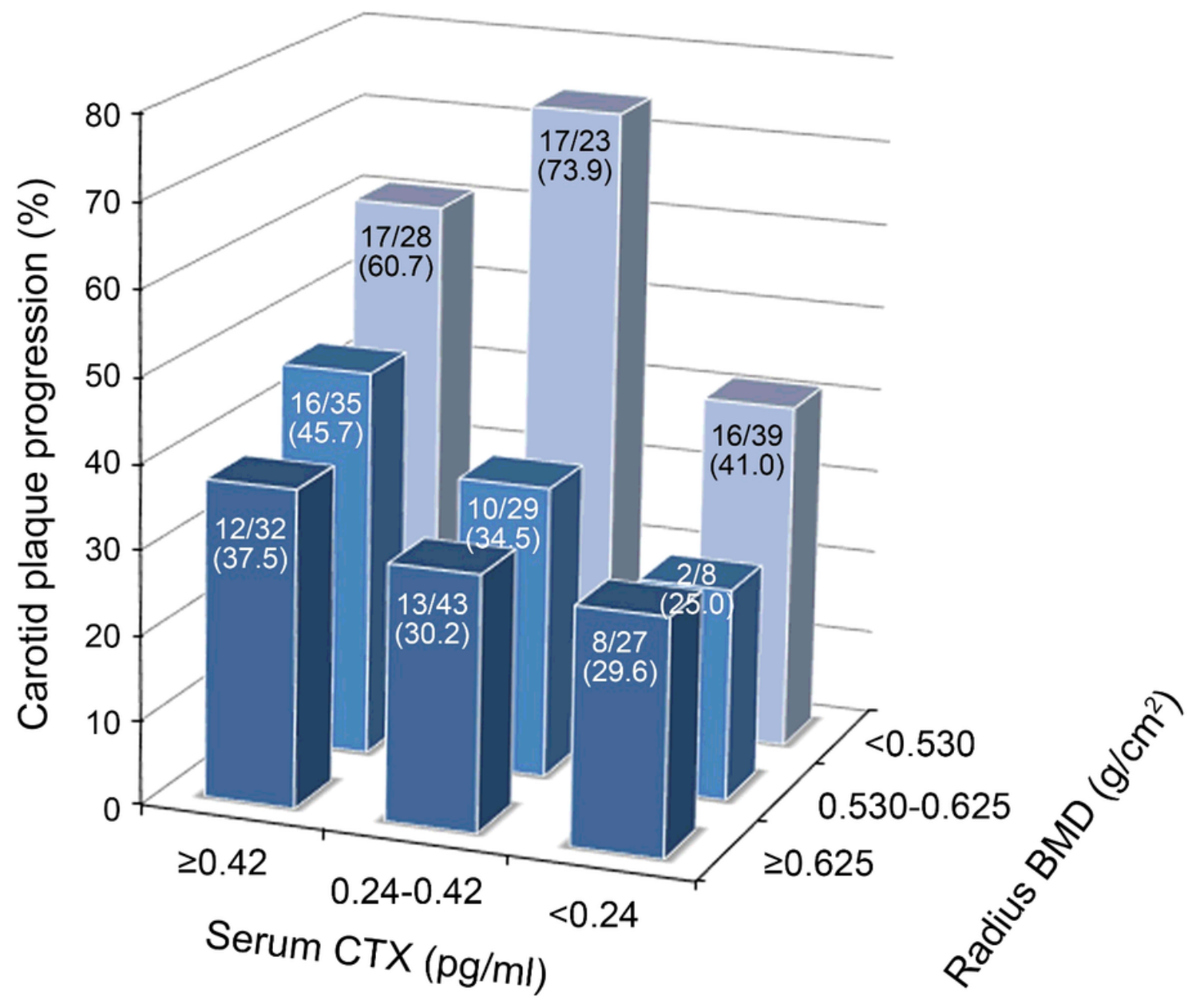

Figure 2

Three-dimensional bar graph showing the interaction of the serum CTX-I level and radius BMD on carotid plaque progression.

\section{Supplementary Files}

This is a list of supplementary files associated with this preprint. Click to download.

- supplementarydata.docx 